Sergio RIESE

\title{
Una nuova specie di Elateridae della Tailandia: Cryptalaus chiangmaiensis n. sp. (Coleoptera, Elateridae)
}

\begin{abstract}
Riassunto - L'autore descrive una nuova specie di Elateridae della Tailandia: Cryptalaus chiangmaiensis n. sp. (ð̊ㅇ) di Doi Pui, Chiang Mai. La nuova specie mostra lo stesso habitus di C. beauchenei (Fleutiaux, 1903), ma risulta nettamente distinta da questa per il pronoto e le elitre nere, coperti da una fitta pubescenza bianca a macchie nere. Viceversa il pronoto di C. beauchenei è rosso, munito di pubescenza breve e rada, le elitre sono nere e coperte da pubescenza nera e sparsa intercalata da tre bande trasversali di pubescenza bianca e fitta.

Abstract - A new species of Elateridae from Thailand: Cryptalaus chiangmaiensis n. sp. (Coleoptera: Elateridae).

A new species of click beetle from Thailand is described: Cryptalaus chiangmaiensis n. sp. (ð̊), loc. typ.: Thailand, Doi Pui, Chiang Mai. It shows the same habitus of C. beauchenei (Fleutiaux, 1903), but from the latter is markedly distinct for the pronotum and elitrae black, covered by a white pubescence with some black spots, while the pronotum of $C$. beauchenei is red, not covered by a very fine pubescence, and elitrae are black, with sparse black pubescence and with three only transversal bands of white pubescence.
\end{abstract}

Key words: Coleoptera, Elateridae, Hemirhipinae, Cryptalaus, new species, Thailand.

\section{INTRODUZIONE}

Ohira (in data maggio 1967) propose il nome Cryptalaus come sottogenere di Alaus Eschscholtz, 1829 per tutte le specie Paleartiche ed Indomalesi; Neboiss (in data luglio 1967) istituì il subgenere $\mathrm{Pa}$ racalais per alcune specie australiane. Ohira (1990) elevò Cryptalaus al rango di genere proponendo $\mathrm{Pa}$ racalais Neboiss come sinonimo essendo stato descritto due mesi dopo. Calder (1996) ha riabilitato Paracalais come genere valido per le specie di $\mathrm{Au}-$ stralia, Nuova Guinea e isole Norfolk.

Bouwer (1993) ha descritto due specie, Paracalais sabinae e $P$. birmanicus, senza tener conto del lavoro di Ohira; propongo quindi due nuove combinazioni: Cryptalaus sabinae Bouwer comb. n. e Cryptalaus birmanicus Bouwer comb. $\mathrm{n}$.

Il materiale esaminato è depositato presso il Museo civico di Storia naturale "G. Doria" di Genova (MCSN), e presso la collezione dell'autore (SRGI)

Misure: la lunghezza è misurata lungo la linea mediana, dal margine anteriore della fronte all'apice dell'elitra; la larghezza è misurata nella parte più larga del corpo; la lunghezza del pronoto, superiore a un terzo di quella delle elitre, è misurata sulla linea mediana, la larghezza alla base, davanti agli angoli posteriori.

\section{DESCRIZIONE DELLA SPECIE}

Cryptalaus chiangmaiensis n. sp. (Fig. 1A, B) Materiale esaminato. Holotypus $\widehat{o}$ (MCSN) e 2 Paratipi $\delta^{\lambda}$ : Thailand, Doi Pui, Chiang Mai, 20 - V
- 1985, S. Steinke cessit. - Paratipi: 1 \} \text { NE Thailand } - 150 Km NE Khon Khaen, Nam Nad n.p., 2-6 - V 2004, J. Louda cessit (SRGI); 1 T Thailand, Doi Pui - Chiang Mai, 2 - VI - 1984, S. Steinke cessit, Alaus sp. - C.M.F. von Hayek det. 1988 (MCSN); 1 q Thailand, Doi Pui - Chiang Mai, 23 - V - 1985, S. Steinke cessit; 2 오 Thailand, Doi Pui - Chiang Mai, 17 - V - 1986, S. Steinke cessit; 2 우 Thailand, Doi Pui Chiang Mai, 3 - VI - 1986, S. Steinke cessit (SRGI).

DesCrizIOne. Dimensioni: $\widehat{\jmath}$ lunghezza 14 - $17 \mathrm{~mm}$., larghezza 3,5 - $4 \mathrm{~mm}$; + lunghezza $15-17 \mathrm{~mm}$, larghezza 4 - $5 \mathrm{~mm}$.

Corpo stretto, cilindrico, convesso; tegumenti lucidi; colore nero, antenne, palpi e zampe neri; corpo ricoperto da una fitta pubescenza bianca che nasconde il colore di fondo, con macchie di pubescenza nera, variabili di estensione; sul pronoto una macchia apicale ed una basale, due discali arrotondate, due lineari, brevi, adiacenti ai lati, che partono dall'apice e non raggiungono la metà della lunghezza del pronoto, due piccole e arrotondate, laterali, vicino alla base; sulle elitre una macchia basale subquadrata attorno allo scutello, sei macchie attigue al bordo laterale: due arrotondate, a circa metà lunghezza, due più piccole a circa un quinto della lunghezza e due preapicali; l'interstria suturale e l'apice sono sempre rivestite di pubescenza bianca e fitta; sul lato ventrale la pubescenza è bianca e uniforme.

Capo con forte depressione centrale, trapezoi- 
dale all'apice, pubescente, con punteggiatura forte, profonda. Antenne più corte del pronoto, dentate dal quarto articolo, pubescenti; secondo articolo globoso, terzo subconico e lungo circa il doppio del secondo, insieme subeguali al quarto, dal quarto al decimo subtriangolari, poco più larghi che lunghi, ultimo ellissoidale, più lungo dei precedenti.

Pronoto convesso, $1,25 \times$ più lungo che largo, allargato in avanti, leggermente sinuato lateralmente, ristretto verso gli angoli posteriori che sono divergenti, carenati; carena ben distinta dal margine laterale, punteggiatura doppia, con punti larghi, rotondi, ombelicati, inframmezzati da punti più piccoli; distanza fra i punti pari al loro diametro sul disco, inferiore ai lati e alla base. Prosterno con punti simili a quelli del pronoto sul lobo prosternale, di diametro inferiore e più ravvicinati sulle propleure. Apofisi prosternale incurvata all'apice. Scutello allungato, stretto, submitrale.

Elitre parallele, ristrette solo all'apice, punteggiate-striate, con due piccole spine terminali divergenti; strie ben incise dalla base all'apice, interstrie convesse, rugose alla base, con punteggiatura fine e sparsa.

Edeago dell'Holotypus come nella Fig. 1B.

Note comparative. C. chiangmaiensis n. sp. si distingue da C. beauchenei (Fleutiaux, 1903), che presenta habitus molto simile, per il corpo totalmente nero, ricoperto di pubescenza bianca, fitta, con alcune macchie nere, mentre $C$. beauchenei ha il pronoto rosso, ricoperto solo da una pubescenza fine e rada, e le elitre nere, con pubescenza nera e sparsa, e solo tre fasce di pubescenza bianca e fitta.

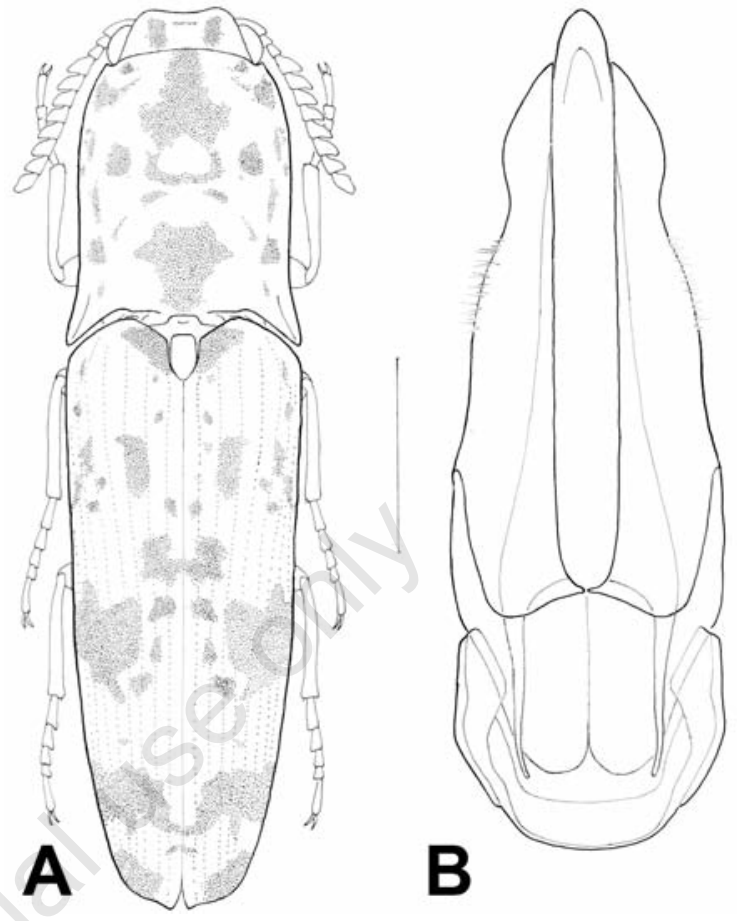

Fig. 1. Cryptalaus chiangmaiensis Riese, Holotypus $\widehat{\delta}$ : A. habitus schematico, B. edeago in visione dorsale.

Derivatio NOMINIS. Il nome deriva dalla località di provenienza.

\section{RINGRAZIAMENTI} disegni.

Si ringrazia l'amico Ivo Gudenzi, autore dei

\section{BIBLIOGRAFIA}

Bouwer R., 1993 - Eine neue Paracalais-Art aus Thailand (Coleoptera, Elateridae) Entomol. Z. 103 ( 1 - 2) 15 - 19.

Bouwer R., 1993 - Eine neue Paracalais-Art aus Burma (Coleoptera, Elateridae) Entomol. Z. 103 (4) 46 - 54.

CALDER A.A., 1996 - Click Beetles: Genera of Australian Elateridae (Coleoptera). Monographs on Invertebrate Taxonomy, Collingwood, CSIRO Publishing, v. $2 \mathrm{X}+401 \mathrm{pp}$.

FleutiauX E., 1903 - Description de deux Elatérides nouveaux (Col). Bulletin de la Socièté entomologique de France, 228 $-229$.

Neboiss A., 1967 - The genera Paracalais gen. nov. and Austrocalais gen. nov. (Coleoptera, Elateridae). Proceedings of the Royal Society of Victoria 80: 259 - 287.

OHIRA H., 1990 - Notes on the genus Paracalais and its allied genera. Gekkan-Mushi, no. 234, pp. 19 - 21.

Indirizzo dell'autore:

Sergio Riese, corso Sardegna 46 - 11d, I-16142 Genova GE, Italia. E-mail sergio.riese@libero.it 